I wish to thank Prof. R. V. Seshaiya for suggesting the problem and guidance, and Prof. A. D. Peacock for reading the manuscript.

Department of Zoology,

JOSEPH $\mathrm{J}_{\mathrm{ACOB}}$

Annamalai University

Annamalainager. July 27.

Jacob, J., Curr. Sci., 23, 56 (1954).

2 Kostoff, D., Genetica, 21, 285 (1939)

'Darlington, C. D., "Recent Advances in Cytology" (2nd edit., 1937).

${ }^{4}$ Suomalainen E., Hereditas, 26, 51. (1940).

\section{Constitution of Cynarine, the Active Principle of the Artichoke}

Ix has been known for some time that the artichoke (Cynara scolymus) contains some hitherto unidentified substances which stimulate biliary secretion and cholesterinic metabolism ${ }^{1}$. The extraction of these substances can be carried out by infusion or decoction. We have been able to isolate from cauline leaves of Cynara scolymus a crystalline substance which we have also found in Cynara cardunculus. Because of its physiological activity, we have used the word 'cynarine', which has been generally used for the chemical principle of the artichoke with the same activity ${ }^{2}$.

We have isolated this compound by treating a decoction of the fresh leaves with lead acetate. The precipitate is extracted with dilute acetic acid and the lead is removed with sulphuric acid and hydrogen sulphide. Crystallization of the cynarine is obtained after concentration and standing. The yield is $0 \cdot 1-0.2$ p.p.m. of the leaves. It gives flat, needles or colourless small plates of melting point $227-228^{\circ}$ (dec.). It is slightly soluble in cool or warm water, more soluble in alcohol and acetic acid.

The substance has a left-handed rotatory power : $[\alpha]^{250},=-59$ (methanol, $c=4$ ). It is a weak acid, giving deep yellow alkaline solutions slightly stable in air. It gives a green colour with ferric chloride; it can be easily oxidized and gives a yellow precipitate with lead acetate and with barium hydroxide.

After drying in air to constant weight the percentage composition is $\mathrm{C}_{25} \mathrm{H}_{24} \mathrm{O}_{12} \cdot \mathrm{H}_{2} \mathrm{O}$. It gives $\mathrm{a}$ hexa-acetyl derivative (m.p. $168-172^{\circ}$ ) and a tetrahydro derivative (m.p. 134-140 ${ }^{\circ}$ ).

Protocatechuic acid (3,4-dihydroxybenzoic acid) can be obtained by fusion with alkali. Its saponification by diluted alkalis produces quinic acid $(1,3,4,5$-tetrahydroxycyclohexane carboxylic acid) and two molecules of caffeic acid (3,4-dihydroxycinnamic acid); saponification of the tetrahydroxy derivative produces quinic acid and dihydrocaffeic acid.

Methylation of cynarine with methyl sulphate and potassium carbonate in acetone gives pentamethylcynarine (m.p. $160-161^{\circ}$ ). Alkaline saponification of this compound gives methyl alcohol, quinic acid and two molecules of 3,4-dimethoxycinnamic acid; permanganic oxidation gives veratric acid.

Such data are sufficient for establishing that cynarine is a three-depside which occurs by esterifica. tion of two molecules of caffeic acid and one of quinic acid.
Because in the synthesis of cynarine the caffeic groups are introduced on the $r$-lactone of quinic acid (quinide) we can exclude, among six isomeric esters, those blocking - $\mathrm{OH}$ of quinic acid in position 3. The pentamethyl-cynarine is stable to periodic acid and for this reason we can exclude the isomer 1-5 also. Hence, we can reduce the number of possible isomers to two (1-4 and 4-5).

The complete methylation of pentamethyl-cynarine by methyl iodide and silver oxide gives a substance that can be saponified. We obtain 3,4-dimethoxycinnamic acid and a dimethylether of quinic acid (m.p. 136-138 ). Because of the resistance of this last compound to oxidation with periodic acid, it must be the 3,5-dimethoxy 1,4-dihydroxycyclohexane carboxylic acid.

Therefore the chemical formula of cynarine is :<smiles>CCCOOC1C(O)CC(OOC(=O)C=CC2CCC(O)C(O)C2)(C(=O)O)CC1O</smiles>

More details of this work will be published elsewhere, with an account of the synthesis of cynarine.

\section{Maria Luisa Scarpati \\ Istituto di Chimica Organica, \\ Università di Poma. July 21. \\ ${ }^{1}$ Cf. Chabrol, E., Charonnat, R., Maximin, M., and Waitz, R., C.R. Soc. Biol., 108, 1020 (1931). 'Tixier, L., and de Sère, S., Chem Abstr., 7535 (1937). Tixier, L., Presse Médicale, 47, 880 (1939). Schoenholzer, G., Schwz. Med. Wschr., 69, 1288 (1939). Roffo, A. H., Bol. Inst. Med. Exp. Cancer, 20, 65 (1943). Legrand, G. C.R. Acad. Sci., Paris, 227, 600 (1948). Hermann, G. R., Chem. \\ ac. Ravina, A., Presse Médicale, 42, 1307 (1934). \\ A New Paper Column for Preparative Chromatography}

Several types of paper columns have been described for separating closely related substances on a preparative scale; but with these methods it has proved difficult to get the same degree of separation as is achieved by simple paper-strip experiments' ${ }^{1}$. This disadvantage is overcome by the present method, the principles of which have been reported in a previous communication ${ }^{2}$. The technique has now been improved and simplified.

The column (manufactured by Grycksbo Pappersbruk, Sweden, and sold by LKB-Produkter Fabriksaktiebolag, Stockholm) consists of a roll of filter paper $(A)$, closely packed around a central core $(B)$ of inert material and placed in a poly. thene tube $(C)$, closed at each end (Fig. 1). Close packing is a prerequisite for sharp fronts, because otherwise the solvent velocity varies through the cross-section of the column, reaching a maximum at the outer and inner column surfaces. Before using the column, solvent must be absorbed by the paper roll, which then swells and, being confined by the walls of the column, is subjected to great pressure. This condition ensures a constant flow velocity throughout the cross-section, thus achieving straight fronts and sharp separations. After the paper roll has been wetted by solvent through the lower cover, tube $G$ is dipped into the 